\title{
Meningovasculitis luética de la médula espinal: "Spinal vascular syphilis of Singer" Luetic meningovasculitis of spinal cord: "Spinal vascular syphilis of Singer"
}

\author{
Carolina Núñez D. ${ }^{1}$, Renato Verdugo L. ${ }^{2}$ y Luis Cartier R. ${ }^{2}$
}

\begin{abstract}
Meningovascular syphilis is the most prevalent form of neurosyphilis. The meningeal compromise begins during the secondary period of the disease and the CSF usually shows inflammatory signs with presence of lymphocytes and increase of gammaglobulins. The VDRL in CSF is highly specific. The inflammatory phenomenon compromises the cerebral and spinal arteries. We analyzed a very singular case of meningovascular syphilis of the spinal cord in 63 year-old men that expressed a left crural monoplegia associated to urinary retention and contralateral sensitive level, preceded of left flank pain (spinal cord angina) that fulfilled the diagnosis criteria of meningovascular syphilis. The MRI of the spinal cord showed enhanced antero-lateral segmental lesion at D7-D8 and cerebral MRI corroborated the presence of asymptomatic syphilitic arteritis with injuries of both thalamus. The patient was treated with endovenous penicillin $G$ and metilprednisolona that showed significant improvement. We conclude that these manifestations of the neurosyphilis, although rare, are still a reality especially in non treated patients.
\end{abstract}

Key words: Neurosyphilis, Meningovascular syphilis, spinal cord infarction. Rev Chil Neuro-Psiquiat 2009; 47 (2): 138-143

\section{Introducción}

$\mathrm{L}$ a lúes meningovascular es la forma de neuro sífilis más prevalente. El compromiso pre$\mathrm{COz}$ de las meninges durante el periodo secundario de la enfermedad hace que la espiroqueta se consolide en esas estructuras, combinando la inflamación meníngea con el daño de los vasos ${ }^{1}$. El compromiso se inicia a las pocas semanas de apa- recer el chancro primario y puede continuar hasta 12 años después de la primoinfección. Se estima que la incidencia de neurosífilis en casos no tratados fluctúa entre 5 y $10 \%{ }^{2}$.

El líquido cefalorraquídeo (LCR) suele ser el principal testigo del fenómeno inflamatorio, mostrando pleocitosis de predominio mononuclear y aumento de las proteínas, en particular de las gammaglobulinas. Las alteraciones licuorales se

Recibido: 15 de mayo de 2009

Aceptado: 1 de junio de 2009

Residente de Neurología. Servicio de Neurología Hospital Salvador, Facultad de Medicina Universidad de Chile.

2 Servicio de Neurología Hospital Salvador. Departamento de Ciencias Neurológicas Facultad de Medicina, Universidad de Chile. 
encuentran en 30 a 50\% de los pacientes sifilíticos sin sintomatología neurológica ${ }^{3}$. En este contexto las pruebas serológicas también son positivas. El VDRL en LCR es el test más utilizado, porque tiene un $99,8 \%$ de especificidad para confirmar el diagnóstico de neurosífilis, aunque conlleva una baja sensibilidad $(30-50 \%)^{4}$.

La sífilis meningovascular se expresa histopatológicamente por infiltrados linfoplasmocitarios de la leptomeninge y cambios arteríticos. En los vasos pequeños toma la forma de la endarteritis de Nissl-Alzheimer; y en los vaso mediano se manifiesta como arteritis de Heubner, que consiste en una progresiva destrucción de las fibras elásticas y musculares de la capa media, que secundariamente generan una reacción fibroblástica subendotelial. Estos cambios arteriales dan origen a lesiones isquémicas o directamente a infartos del cerebro o de la médula espinal ${ }^{5}$.

En el cerebro las áreas preferenciales del daño vascular, se encuentran en el territorio distal de las arterias lenticuloestriadas. Resultando características las lesiones de los ganglios basales que afectan también la capsula interna. Sin embargo, muchas de estas lesiones son asintomáticas. También se lesiona el centro semioval en áreas paraventriculares que se manifiesta por pequeñas y múltiples lesiones no contiguas ${ }^{6}$.

En 1882 Bastian, en el Quain's dictionary of medicine, fue quien primero sugirió que el compromiso sifilítico de la médula espinal se debía a trombosis de los vasos espinales. En 1894 Williamson reportó un caso estudiado con anatomía patológica, que le permitió remarcar la importancia de los accidentes vasculares en estos pacientes. El trabajo de Singer publicado en 1902, con la revisión de 15 pacientes sifilíticos con daño medular y estudio necrópsico, demostró que las lesiones de la médula espinal se debían a trombosis de los vasos espinales por arteritis específica. El título de la publicación: "Pathology of so-called acute myelitis", hace referencia al error conceptual que se tenía de la sífilis de la médula espinal ${ }^{7}$.

Adams y Merritt en 1944, estudiaron 32 casos de sífilis con compromiso medular, surgidos de entre 2.231 pacientes sifilíticos. En ese trabajo ellos intentaron clasificar las patologías sifilíticas de la médula definiendo cuatro grupos: A) $\mathrm{Me}$ ningomielitis sifilitica que incluye entre otros la paraplejia de Erb y la meningomielitis amiotrófica de Martin; B) Sifilis vascular espinal de Singer; C) Goma sifilitico y Paquimenigitis sifilitica (Joffroy y Charcot) y D) Poliomielitis sifilitica de Gowers. Diez pacientes de esta serie desarrollaron una paraplejia aguda, con nivel sensitivo dorsal y pérdida del control esfinteriano, que concordaban con el concepto de sífilis vascular espinal descrita por Singer. Dentro de la serie representaron el $0,44 \%$ de los casos de sífilis y sólo $1 / 3$ de la sífilis medular8.

Queremos comunicar un caso muy singular de sífilis meningovascular de la médula espinal.

\section{Caso Clínico}

Hombre de 63 años, con el antecedente de contactos sexuales sin protección y de haber presentado lesiones genitales autolimitadas. Consultó por un cuadro que se inició con intenso dolor del flanco izquierdo, distensión abdominal y sensación nauseosa que cedió al tercer día, coincidiendo con la aparición de debilidad de la pierna izquierda, que rápidamente llegó a una monoplejia crural, acompañada de vejiga neurogénica.

A su ingreso al Servicio de Neurología del Hospital del Salvador. El examen mental y de pares craneales era normal. Presentaba una plejia de la pierna izquierda, sólo persistía leve contracción del cuádriceps y del adductor, con hipotonía. Tenía hiperreflexia en ambas extremidades inferiores, con reflejo plantar extensor a izquierda y flexor a derecha. Mostraba a derecha una hipoestesia termo-algésica crural, con nivel sensitivo que llegaba a T10. La sensibilidad profunda se encontraba conservada en ambos lados. En el examen físico general llamaba la atención la presencia de múltiples lesiones de aspecto purpúrico en tórax, abdomen y dorso de ambos pies. La tomografía axial computada (TAC) de cerebro no mostró lesiones. El LCR era claro con 102 células a predominio linfocitario, glucosa y proteínas 


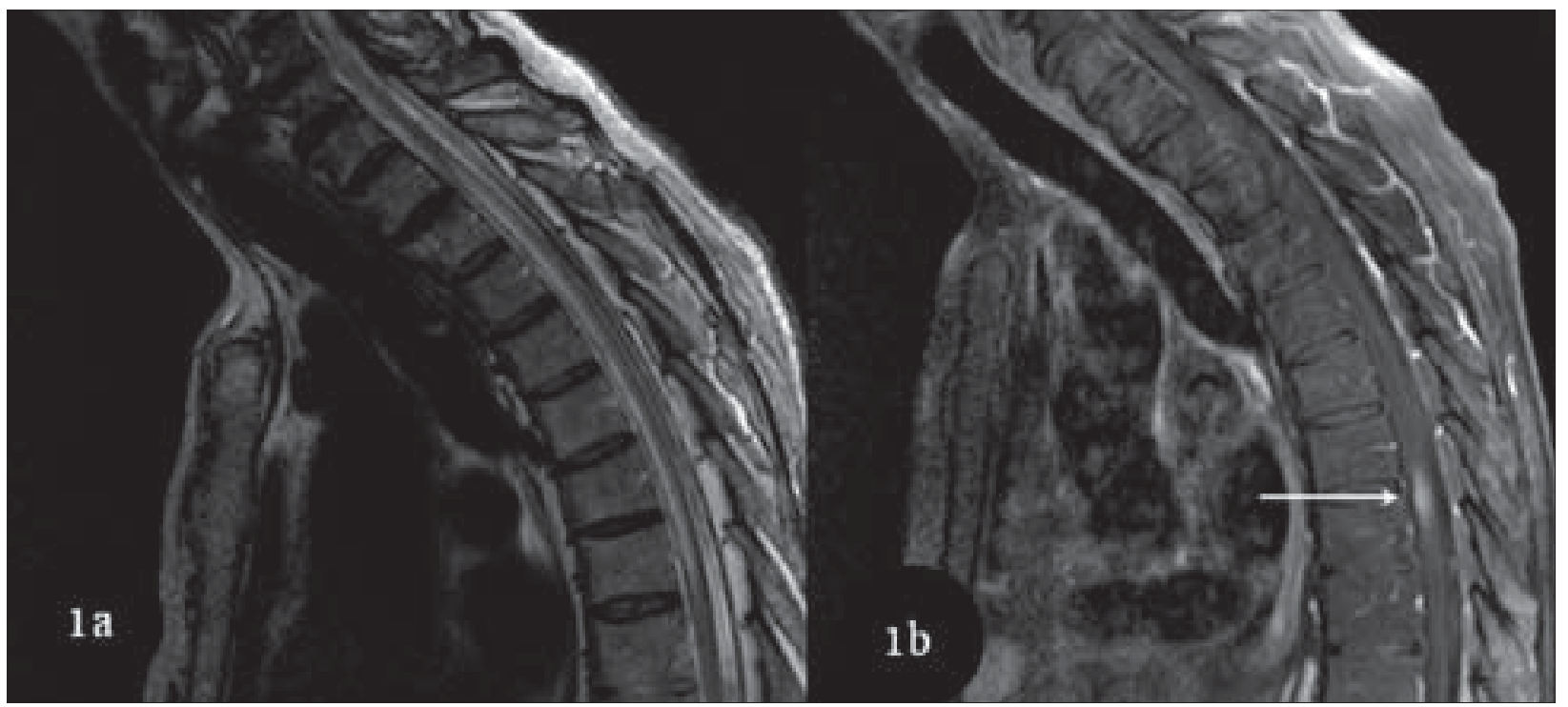

Figura 1. RM de columna dorsolumbar: (a) Sección sagital potenciada en T2 que muestra aumento de señal de la médula espinal desde D1 hasta D11. (b) Sección sagital potenciada en T1 gadolinio con captación de contraste a nivel D7-D8.

normales. IgG elevada y bandas oligoclonales positivas. El estudio serológico de VDRL fue reactivo $1 / 8$ y en el LCR reactivo $1 / 4$. Fueron negativos el HIV y HTLV-1. Los anticuerpos antinucleares fueron positivos 1/80 (patrón homogéneo). Estaban negativos el anti-DNA, anti-cardiolipinas, anti-antígenos nucleares extractables (ENA) y el factor reumatoide. El hemograma y el complemento fueron normales, también el test de Schirmer. La resonancia magnética (RM) de la médula mostró un aumento tenue de la señal en la región centro medular desde D1 hasta D11 (Figura 1a, 1b) y una lesión con captación de contraste a nivel D7-D8 en la hemimédula izquierda (Figura 2). En la RM del cerebro se encontraron focos con incremento de la señal en la sustancia blanca protuberancial y subcorticales bifrontales, además focos de hiperintensidad en ambos tálamos, que mostraban restricción en la difusión sin realce con la administración de contraste (Figura 3$)$.

Se inició tratamiento con metilprednisolona $1 \mathrm{~g}$ endovenoso diario por cuatro días, asociado a penicilina $G$ sódica endovenosa en dosis de 20 millones de unidades diarias por 14 días, seguido por penicilina-benzatina 2,4 millones de unidades intramuscular semanal por tres semanas. Las lesiones cutáneas desaparecieron precozmente durante el tratamiento, logrando bipedestación y marcha con apoyo al finalizar la terapia.

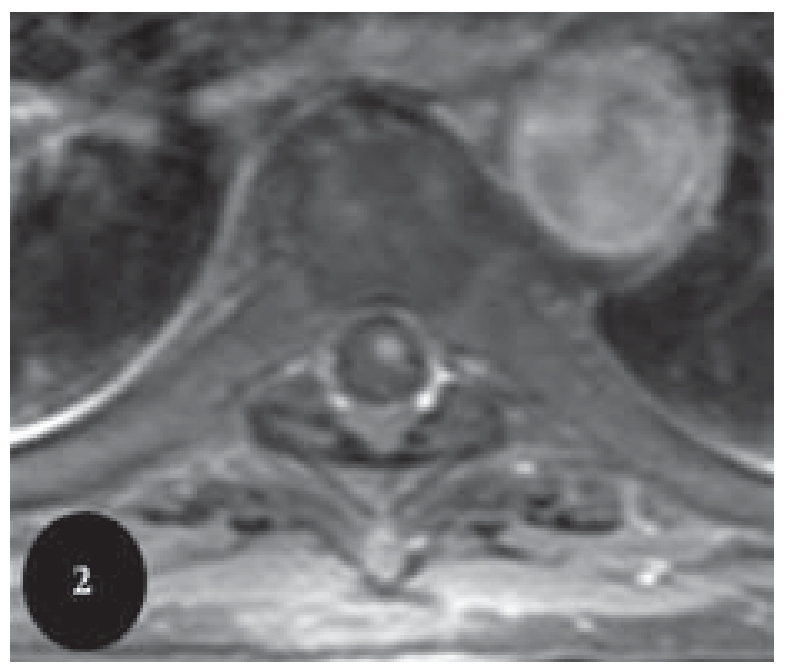

Figura 2. RM de columna dorsolumbar: Sección axial potenciada en T1 gadolinio que muestra captación anterolateral izquierda, concordante con la clínica. 


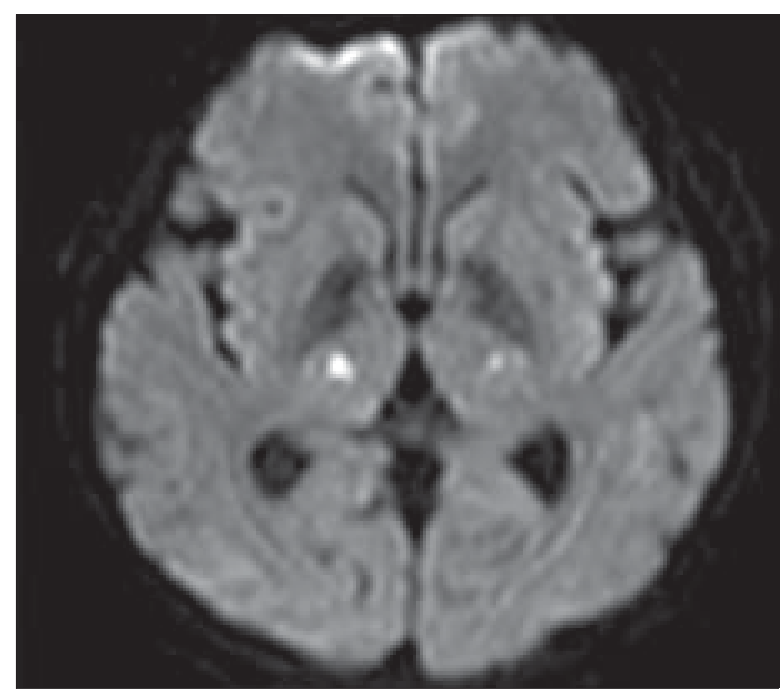

Figura 3. RM de cerebro difusión: Hiperintensidad focal de ambos tálamos que presentan restricción de la difusión. .

La RM de control practicada dos meses después de iniciado el episodio vascular, mostraron una mejoría significativa. Aunque en D7 y D8 persistía menor hiperintesidad en el área lesionada (Figura 4).

\section{Discusión}

Hemos analizado un paciente de 63 años, portador de una monoplejia crural causada por una sífilis meningovascular. Las lesiones de la médula espinal son infrecuentes, y lo son más las de origen sifilítico, incluso lo eran antes de la penicili$\mathrm{na}^{4}$. Además este caso resulta excepcional, porque la lesión compromete el territorio de una arteriola penetrante de la médula y en la RM del encéfalo presenta lesiones arteriolares múltiples sin expresión clínica, que demostrarían un compromiso de los pequeños vasos, que hacen revivir la "endarteritis de Nissl-Alzheimer". Además en este paciente se confirma el concepto de Adams de la inexistencia de formas de sífilis espinal aisladas, especialmente cuando se afectan los pequeños vasos ${ }^{5}$.

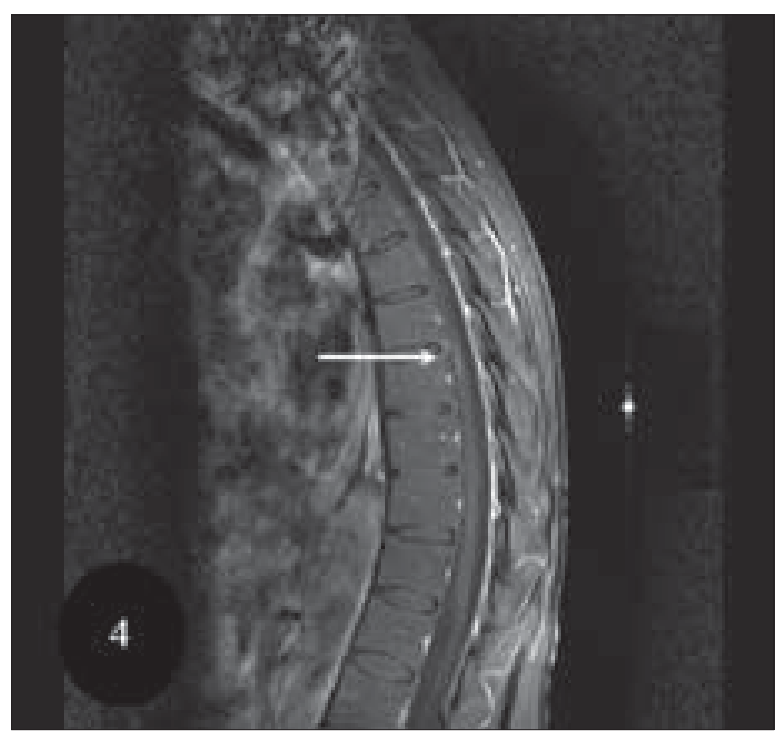

Figura 4. RM a los dos meses de evolución: Sección sagital potenciada en $\mathrm{T} 1$ gadolinio con pequeño foco de captación en el nivel D7-D8 posterior al tratamiento.

Como se sabe la arteria espinal anterior solventa la irrigación de los dos tercios anteriores de la médula, el tercio posterior es tributario de las arterias espinales posteriores. Cada segmento antero-central esta irrigado por las arterias penetrantes sulcales que se originan en la arteria espinal anterior. Surgen a derecha e izquierda alternadamente y en distinto nivel, irrigando la mitad del segmento medular correspondiente ${ }^{10}$. Una de esas pequeñas arterias fue la comprometida en nuestro paciente, lesionando sólo los dos tercios anteriores del lado izquierdo del segmento D7-D8, como muestra la Figura 2.

Clásicamente la isquemia de la arteria espinal anterior produce una lesión bilateral de las astas anteriores, del haz espinotalámico anterior y lateral y del tracto corticoespinal bilateral. Generando una para o tetraparesia, vejiga neurogénica y compromiso sensitivo disociado, con anestesia termoalgésica por debajo de la lesión ${ }^{11}$, que no fue el caso de nuestro especial paciente

En 1986 Napolitano N. y cols, estudiaron 8 casos de infarto de la médula espinal de etiología primaria vascular, la lesión del segmento dorsolumbar estuvo presente en 6 pacientes, como en 
la mayoría de las series. El dolor superficial y profundo del territorio segmentario afectado y/o dolor lumbar, fue el síntoma premonitorio más destacado en la isquemia medular y que precedió en horas o días la paraplejia en 7 de ellos. Nuestro enfermo también presentó dolor intenso en el flanco izquierdo como síntoma precoz de su monoplejia. En la serie de Napolitano el estudio de LCR fue anormal en 5/8 pacientes, que mostraban hiperproteinorraquia. La TAC de médula realizada en 6 de los pacientes fue normal ${ }^{10}$. Demostrando la poca utilidad de este examen, como en nuestro caso.

Weidauer S. y cols, en el año 2002 estudiaron con RM 16 casos de accidente vascular de la médula. Quince presentaban compromiso de la arteria espinal anterior. En todos se observó que la lesión tenía hiperintensidad en cortes sagitales en secuencia T2 y se asociaba a edema medular. En dos pacientes que la RM fue negativa en las primeras 3 horas de iniciados los síntomas, se confirmó el infarto al 5to y 8vo día respectivamente. También encontraron imágenes de "snake eyes" (ojos de serpiente) o "owl's eyes" (ojos de búho) por hiperintensidad bilateral de las astas anteriores de la médula en secuencia T2, como típicos de una lesión isquémica ${ }^{11}$. Por su parte, Chilver y col, 2009, reconocieron como infrecuente la arteritis sifilítica de la medula y señalaron signos en la RM que permitirían separarlos de otras causas. En cortes sagitales en secuencia T2 observan una prolongación hiperintensa en punta de lápiz en la parte central de la médula, parecida a la imagen observada en nuestro pa- ciente (Figura 1). También observaron captaciones anormales en áreas superficiales de la médula, semejantes al escurrimiento de gotas de cera de una vela, que se hacen más evidentes con el uso de gadolinio ${ }^{9,11}$.

Se ha postulado también como elemento diferenciador de otros procesos medulares, que los infartos tienen un territorio vascular que las inflamaciones (mielitis) no respetan comprometiendo más de dos tercios de la médula en sentido transversal ${ }^{13}$. Sin embargo, concluyen que las imágenes hiperintensas descritas en T2 podrían observarse también en enfermedades inflamatorias $^{11,13}$

Un elemento clínico diferencial puede ser el antecedente de síntomas deficitarios de inicio agudo que apoyarían la etiología isquémica. Eventualmente la repetición de imágenes en lesiones vasculares puede mostrar transformación hemorrágica en algunos pacientes. Existen pocos casos de infartos medulares diagnosticados con técnica de difusión. Este método puede incrementar la sensibilidad del diagnóstico y ayudar en el diagnóstico diferencial de cuadros inflamato$\operatorname{rios}^{8}$.

La confirmación del diagnóstico de meningovasculitis luética debe basarse en los criterios recomendados por el US Centers for Disease Control and Prevention (CDC) que incluye sintomatología neurológica, LCR con pleocitosis y VDRL en LCR y suero reactivos ${ }^{4}$.

Nuestro paciente presentó la clínica de un infarto medular que cumple con los requisitos de neurolúes.

\section{Resumen}

La sifilis meningovascular es la forma de neurosifilis más prevalente. El compromiso meníngeo se inicia durante el período secundario de la enfermedad y el LCR suele mostrar signos inflamatorios con presencia de linfocitos y aumento de gammaglobulinas. El VDRL es altamente específico en LCR. El fenómeno inflamatorio compromete las arterias cerebro-espinales causantes del daño en el SNC. Se analiza un caso de sifilis de la médula espinal, en un hombre de 63 años, que se inicia con dolor del flanco izquierdo (angina medular), que continuó con una monoplejia crural izquierda, asociada a vejiga neurogénica y nivel sensitivo contralateral. 
Cumplía con los criterios diagnósticos de sífilis meningovascular. La RM de la médula espinal mostró una lesión segmentaria en la zona antero-lateral izquierda en D7-D8 y la RM cerebral corroboró la presencia de una arteritis sifilítica con lesiones asintomática en ambos tálamos. Fue tratado con penicilina $G$ endovenosa y metilprednisolona que lo mejoraron significativamente. Se concluye que estas manifestaciones de neurolúes en la médula espinal, aunque raras, siguen vigentes especialmente en paciente no tratados.

Palabras clave: Neurosifilis, Sífilis meningovascular, Infarto de la médula espinal.

\section{Referencias}

1. Conde-Sendin M A, Hernández-Fleta J L, Cárdenas-Santana M A, Amela-Peris R. Neurosífilis: formas de presentación y manejo clínico. Rev Neurol 2002; 35 (4): 380-386.

2. Tsui E Y K, Ng S H, Chow L, Lai K F, Fong D, Chan J H M. Syphilitic myelitis with diffuse spinal cord abnormality on MR imaging. Eur Radiol 2002; 12: 2973-2976.

3. Marra C, Maxwell C, Smith S, Lukehart S, Rompalo A, Eaton M, et al. Cerebroespinal fluid abnormalities in patients with syphilis: Association with clinical and laboratory features. The Journal of Infectious Diseases 2004; 189: 369-376.

4. O'Donnell J, Emery C. Neurosyphilis: A Current Review. Current Infectious Disease Reports 2005; 7: 277-284.

5. Adams R D, Merrit H. Meningeal and vascular syphilis of the spinal cord. Medicine (Baltimore) 1944; 23: 181-121.

6. Adams R D, Victor M, Ropper A. Infecciones del sistema nervioso. en: Principios de neurología. Edit. Adams R.D, Victor M, Ropper. México: Mc GrawHill 1999; 32: 627-632.

Correspondencia:

Luis Cartier R.

E-mail: lcartier@uchile.cl
7. Lowenstein D, Mills C, Simon R. Acute syphilitic transverse myelitis: unusual presentation of meningovascular syphilis. Genitorum Med 1987; 63: 333-338.

8. Silber M H. Syphilitic myelopathy. Genirotum Med 1989; 65: 338-341.

9. Chilver L, Fisher U, Hauf M, Fux C A, Sturzenegger M. Syphilitic Myelitis: Rare, nonespecific, but treatable. Neurology 2009; 72: 673-675.

10. Napolitano E, Cartier L, Ferrer S. Accidente vascular isquémico de la medula espinal. Presentación clínica de ocho casos, dos con estudio anatomopatológico. Rev Chil Neuro-Psiquiat 1986; 24: 119-126

11. Weidauer S, Nichtweiss M, Lanfermann H, Zanella F. Spinal cord infarction: MR imaging and clinical features in 16 cases. Neuroradiology 2002; 44: 851857.

12. Ho Choi K, Soo Lee K, Ok Chung S, Mee Park J, Joo Kim Y, Sook Kim H. Idiopathic Transverse Myelitis: MR Characteristics. AJNR Am J Neuroradiol 1996; 17: 1151-1160.

13. Zhang J, Huan Y, Qian Y, Sun L, Ge Y, MB. Multishot diffusion- weighted imaging features in spinal cord infarction. Spinal Disord Tech 2005; 18 (3): $277-282$ 